\title{
Developing a Convenient Balance Beam for Unknown Loads
}

\author{
Keon Young Yi* Associate Professor \\ Yong Jun Kim* Graduate student
}

\author{
*Dept. of Electrical Engineering \\ Kwangwoon University \\ Seoul 139-701, Korea
}

\author{
Sam Yong Chung** Team Leader \\ Song Soo Han** Senior Researcher/Ph.D \\ Sang Heon Lee** Senior Researcher/Ph.D \\ **Research Institute of Technology, \\ Samsung Corporation \\ 270-1 Seohyun-dong, Bundang-gu, \\ Sungnam-si, Gyonggi-do, 463-824 Korea
}

\author{
Telephone : +822-940-5142 \\ Fax : +822-942-0107 \\ E-mail : keonyi@daisy.kw.ac.kr
}

\begin{abstract}
Balance beam, a controller that is built adopting the gyro effect, can freely control the attitude of an unstructured object by means of the position controlling of an inner gimbal. However in the former research, the weight (inertia) of a load should be known to issue a proper command for the gimbal, which makes it hard to operate the balance beam in field applications because the load can be changed frequently and, moreover, the inertia of the same load could be different according to how the load is held. Therefore an accident could be caused by the operation with an improper gimbal command.

One of the possible approaches to solve the problem is to give a balance beam controller an ability to detect the inertia of a load so that it can limit the velocity of the load ordered by a user. In this paper, we designed a controller that can estimate the inertia of the load based on the result we did before; the fact that when there is smaller inertia of load, the larger amount of the restoration displacement occurs. Thus, the load could be identified by issuing a predefined command to measure the amount of the restoration displacement, which makes us able to construct the controller that can limit the angular velocity of the load by planning the motion. Experimental results show the performance of the controller with some loads.
\end{abstract}

Keywords: gyro effect, balance beam controller, construction equipment, load estimation, gimbal

\section{INTRODUCTION}

In this paper, we designed the balance beam controller which can control unknown load using the CMG (control moment gyro) subsystem. Usually, gyro consists of a wheel spinning at high speed. It has a tendency to maintain its spin axis in a fixed direction in space. A torque exerted about any axis other than the spin axis produces a rotation about the axis that is orthogonal to the applied-torque axis[1][2]. We designed and implemented CGM subsystem for balance beam which has a rotating wheel at high speed and outer gimbal connected to the motor. As the attitude of the outer gimbal changes, rotation force occurs about the vertical axis if the balance beam is mounted on the object in air. We use the torque produced to control the attitude of the control object.

The CMG characteristics are addressed in a few researches such as the stabilizer for gondola and aircraft. Hiroshi KANKI and et al.[3] developed
CMG active vibration control device for gondola to reduce random wind force. And, Ahmed and et al.[4] studied on the gyro pendulum to eliminate the effects of mass imbalance in rotating bodies. Attitude control of spacecraft was addressed by $\mathrm{Li}$ and et al.[5]. Except for KANKI, all these studies have not addressed on an attitude control but on a stabilization of the spacecraft.

In our previous study, we expanded the application area of CMG subsystem from attitude control of the objects only to its stabilization in space. Furthermore, we designed an attitude controller of gimbal with gyro actuator that used wireless communication for actual application in field. However, in the unknown load inertia case, it is not easy even for skilled workers to operate a balance beam. That is caused by the difficulty in estimating the load inertia. If the excessive gimbal operation takes place with wrong estimations, accidents are likely to happen.

In this paper, we attached angular velocity sensor to gyro actuator to stabilize the attitude control of balance beam. Next, we estimated the inertia of load 
using the output of angular velocity sensor and simple manipulation. Using the developed controller that can control the angular velocity of the balance beam, we make it easy to drive and strengthen the stabilization of the previous controller.

\section{BALANCE BEAM}

In this chapter, we will explain what the gyro effect is and how to build the balance beam. We will also briefly introduce the mathematical methods of the balance beam.

\subsection{Gyro effect}

In Figure 1, $L_{x}$ represents angular momentum about x-axis, $N_{y}$ and $J_{\omega}$ stand for torque about y-axis and the inertia of wheel, respectively.

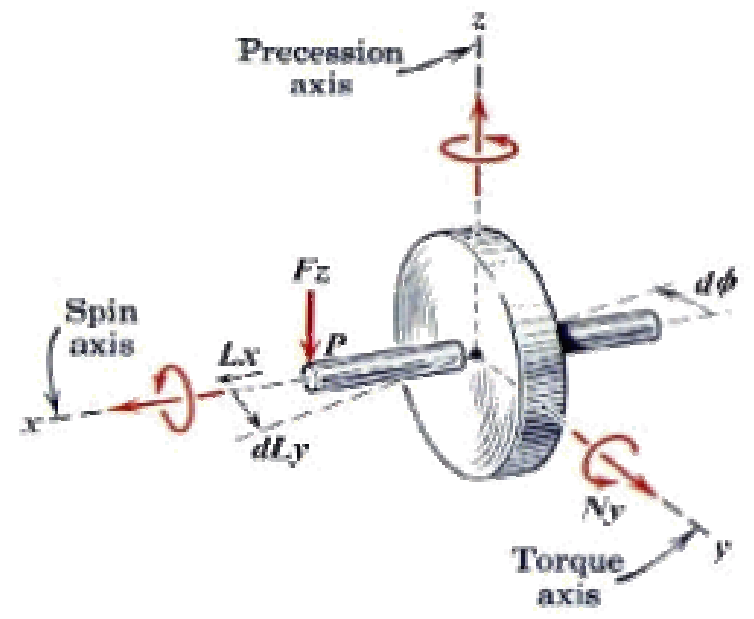

Figure 1. Gyro dynamics

Exerting force at arbitrary point $\mathrm{P}$, we get a torque about y-axis, $N_{y}$, as bellow,

$$
\frac{d L_{y}}{d t}=N_{y}
$$

and we get a changed angular momentum

$$
L=L_{x}+d L_{y}
$$

Since a change of angular momentum, $d L_{y}$, is smaller than $L_{x}$

$$
d \phi=\frac{d L_{y}}{L_{x}}
$$

Substituting (1) into (3), we get

$$
\frac{d \phi}{d t}=\frac{N_{y}}{L_{x}}
$$

From this equation, one can see that rotational motion about the vertical axis, $\mathrm{z}$, could be obtained by applying torque about y-axis.[1][2]

The other, rotational motion torque about horizontal axis, y, is obtained by gimbal motor. At the same time, $\phi$, the rotation of load obtained by vertical axis, $\mathrm{z}$.

\subsection{CMG subsystem}

In this section, the structure of the CMG subsystem, modeling of the system, the controller for the gimbal motor and the wheel motor are explained.

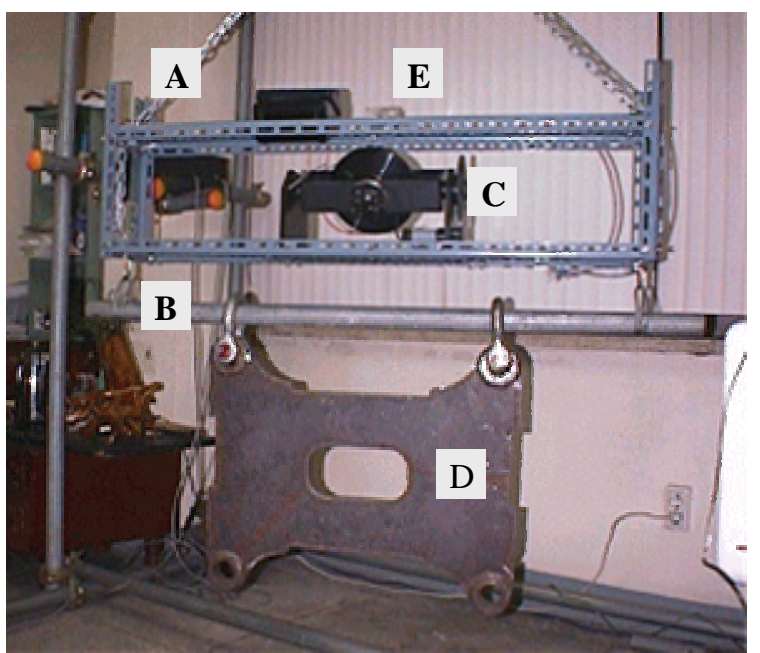

Figure 2. A balance beam system and load

The fabricated CMG subsystem in laboratory scale is shown in Figure 2, and enlarged gyro actuator is shown in Figure 3.

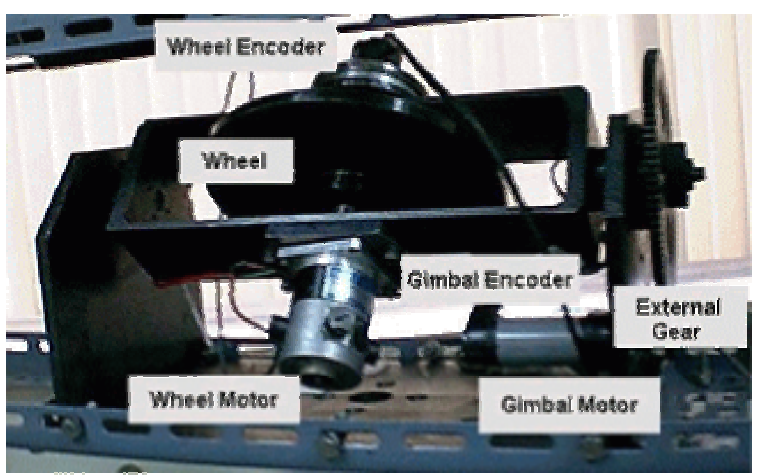

Figure 3. The structure of CMG subsystem

As shown in Figure 2 and 3, the control system consists of a wheel spinning by a motor, inner gimbal supporting the wheel and the wheel motor that is fixed to inner gimbal, and outer gimbal supporting the inner gimbal and gimbal motor that is fixed to outer gimbal. Inner gimbal position can be controlled by the gimbal motor. As shown in Fugure 2, CMG subsystem is suspended from a structure through a swivel hook that has little friction.

The structure (B) to be hung the load (D) is shown 
in Figure 2, and the details of CMG subsystem (C) is shown in Figure 3. The CMG subsystem consists of a high speed wheel and a gimbal that can control the attitude of the balance beam by means of adjusting the wheel rotation axis.

The piezoelectric vibrating gyroscope sensor is shown in Figure 2 (E). It can measure the load inertia using measurements of rotation velocities of the load. Also, a motor and an encoder are attached to both the axis of the wheel and the axis of gimbal.

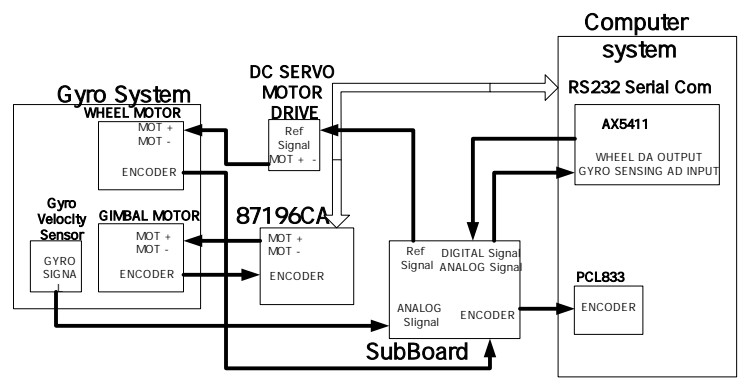

Figure 4. System block diagram

Subsystem block diagram in Figure 2 is shown in Figure 4. The control system consists of a 32-bit computer, Pentium-133MHz, an AX5411 A/D-D/A converter by Axiom Co., PLC833 3-axis quadrature encoder by Adventech, and an amplifier working in torque mode which ha been built with power driver module, 12A8E, 5A5 by Advanced Motion Control. One encoder is used to measure the angular velocity of wheel, and the other encoder is used to measure inner gimbal position and velocity (500pulse through 18:1 gear reduction, and 4:1 external gear). Because the encoder body which measures angular velocity of the wheel can not be fixed firmly to wheel shaft, some noise is made. We solved this problem using second order butterworth filter in CMG control program. The wheel motor is 60[W] DC motor by Tamagawa seiki and gimbal motor is $15[\mathrm{~W}] \mathrm{DC}$ motor by Maxon.

Also, we attached piezoelectric vibrating gyroscope sensor for sensing the angular velocity of load. 12bits digital value that is changed by AX5411 A/D$\mathrm{D} / \mathrm{A}$ converter installed in computer could be measured by that sensor. The attitude of gimbal motor is controlled by means of the LMD18200. Control Board, 87C196 that is developed in our laboratory can control the attidude of load.

The specifications of the major parts organization of the system are listed in Table 1 . The inertia of the balance beam is obtained by assumption that the frame and CMG are regarded as a rectangular parallelepiped of which mass has been distributed evenly.

\subsection{CMG subsystem modeling}

We derive the modeling of CMG subsystem from assumptions that gimbal is lighter than wheel (weight of the gimbal can be regarded as load), a viscous- friction of swivel hook and bearing is very small, and motor inductance is negligible.

Table 1 . The parameters of the system

\begin{tabular}{|c||l|}
\hline Device & \multicolumn{1}{|c|}{ Specification } \\
\hline \hline Wheel Motor & $\begin{array}{l}\text { Tamagawa TRE PMDC 60[W], } \\
\text { 1000p/r Encoder }\end{array}$ \\
\hline Gimbal Motor & $\begin{array}{l}\text { Maxon Amax110857 DC 15[W], } \\
\text { 500p/r Encoder, 72:1 Reduction }\end{array}$ \\
\hline $\begin{array}{c}\text { Wheel mass } \\
\text { /diameter }\end{array}$ & 2.69[kg], 199.9[mm] diameter \\
\hline $\begin{array}{c}\text { CMG subsystem } \\
\text { mass }\end{array}$ & 24.55[kg] \\
\hline $\begin{array}{c}\text { Frame mass and } \\
\text { dimension }\end{array}$ & $\begin{array}{l}\text { 9.3[kg], } \\
789 x 200 x 200[\mathrm{~mm}]\end{array}$ \\
\hline $\begin{array}{c}\text { Balance beam } \\
\text { inertia }\end{array}$ & $1.86\left[\mathrm{kgm}{ }^{2}\right]$ \\
\hline Main Controller & 87c196CA Control Board \\
\hline
\end{tabular}

The transfer function from the voltage applied to the angle of the gimbal motor is

$$
\begin{aligned}
& G_{1}(s)=\frac{\theta_{g c}(s)}{v_{a}(s)}=\frac{k_{m}}{s\left(\tau_{m} s+1\right)} \\
& k_{m}=\frac{k_{1}}{\left(k_{k} k_{b}+f_{m} R_{a}\right)} \\
& \tau_{m}=\frac{\left(J_{g r}+J_{c}\right) R_{a}}{\left(k_{k} k_{b}+f_{m} R_{a}\right)}
\end{aligned}
$$

Where, $k_{b}, k_{t}$, and $\tau_{m}$ are back-emf constant, torque constant, and time constant, respectively [8]. $R_{a}$ stands for armature resistance, $J_{g r}$ represents rotor inertia of gimbal motor, $J_{c}$ for the wheel inertia of circular disk turning about its radial axis.

Then, we assume that the gimbal is lighter than wheel. We considered the case which the load is attached to the gyro actuator.

The relationship between the attitude of the inner gimbal, $\theta_{g c}$, and rotation about vertical axis, $\phi$, is

$$
\begin{gathered}
\left(J_{g r}+J_{c}\right) \frac{d^{2} \theta_{g c}}{d t^{2}}+f_{m} \frac{d \theta_{g c}}{d t}=J_{w} \omega_{w} \frac{d \phi}{d t}-J_{l} \frac{d^{2} \phi}{d t^{2}} \\
J_{w}=M_{w} \frac{r^{2}}{2} \\
J_{l}=M_{l} \frac{\left(a^{2}+b^{2}\right)}{12} \\
J_{c}=M_{w} \frac{r^{2}}{4}+M_{w} \frac{h^{2}}{12}
\end{gathered}
$$

Where, $M_{w}$ and $M_{l}$ are mass of wheel and mass of load, respectively. $r, h, a$ and $b$ represent radius of wheel, thickness of wheel, length of load, and width of load, respectively. $J_{w}$ means wheel inertia about spin axis. Then, transfer function $G_{2}(\mathrm{~s})$ is 


$$
G_{2}(s)=\frac{\phi(s)}{\theta_{g c}(s)}=-\frac{\left(J_{g r}+J_{c}\right) s+f_{m}}{J_{l} s-J_{w} \omega_{w}}
$$

Transfer function of the overall system $G(s)$ is obtained from (5) and (7) as following:

$$
\begin{aligned}
G(s) & =-G_{1}(s) G_{2}(s) \\
& =\frac{1}{s} \frac{\left(J_{g r}+J_{c}\right) k_{m} s+k_{m} f_{m}}{J_{l} \tau_{m} s^{2}+\left(J_{l} s-J_{w} \omega_{w} \tau_{m}\right) s-J_{w} \omega_{w}} \\
& =\frac{1}{s} \frac{b_{1} s+b_{0}}{s^{2}+a_{1} s-a_{0}}
\end{aligned}
$$

Where, $a_{0}, a_{1}, b_{0}, b_{1}$ are determined by the motor parameters and the shape and angular velocity of the wheel. Notice that $a_{1}$ depends on the load inertia, and transfer function has an unstable pole.

\section{BALANCE BEAM CONTROLLER}

In this chapter, we will explain what the restoration of a gimbal is and how to use it to figure out the inertia of an unknown load. Furthermore, we will present a controller incorporating the restoration that can control the angular velocity of an unknown load by adjusting the proper gimbal command.

\subsection{Restoration angle of gimbal}

When we issue the gimbal motion command, the load rotates according to the amount of the command. However, if we cut the power of the gimbal, which initiates the stabilizing mode of the balance beam, it goes backward to stop the load. At this time we define the restoration angle as the difference between the issued gimbal angle and the angle when the load stops.

Here we investigate the relationship between the restoration angle and the load.

The loads, iron plate shown in Figure 2, considered here are summarized in Table 2, where the inertia values in the parentheses are the values including the inertia of the balance beam.

Table 2. Weight and inertia of load

\begin{tabular}{|c||c|c|}
\hline Load & Weight $[\mathrm{kg}]$ & Inertia $\left[\mathrm{kgm}^{2}\right]$ \\
\hline \hline No load & 0 & $0.00(1.86)$ \\
\hline Load 1 & 55 & $0.74(2.70)$ \\
\hline Load 2 & 55 & $2.12(4.12)$ \\
\hline
\end{tabular}

Actually, loads are same, but the hanging direction is different; one is crosswise and the other is lengthwise. The motions of different loads to the gimbal command, 10 degrees, are depicted in Figure 5.
Note that the line marked as load represents the angular velocity of the load measured using the rate gyro sensor built in our lab [6]. The signal seems too noisy since the balance beam was chattering caused by the wheel noise. Actually, the sensor information is not involved in the controller, but it is just a monitoring signal to check the load angular velocity in this study.

In Figure 5, we can see the restoration angles (A, B, C) for different loads, which are getting smaller as the load increasing. Note that the figure (c) is enlarged for the purpose of the comparison. The detailed results are summarized in Table 3.

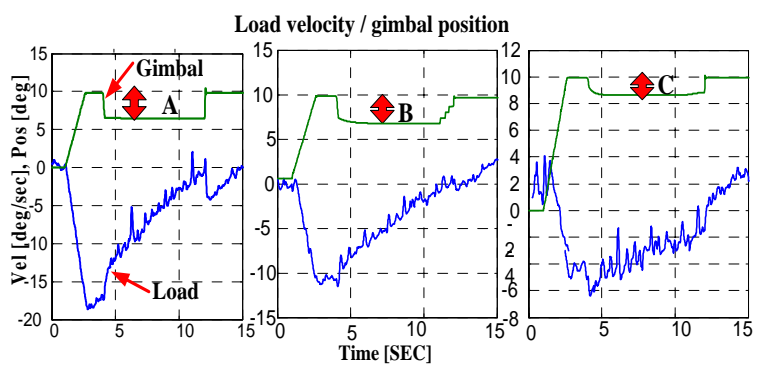
(a): No Load
(b): Load 1
(c) Load 2

Fig. 5 The load angular velocity and gimbal trajectory

Table 3. Gimbal restoration angle for loads

\begin{tabular}{|c|c|c|}
\hline Load & Gimbal command & Restoration angle \\
\hline \hline No Load & \multirow{3}{*}{$10^{\circ}$} & 3.27 \\
\cline { 1 - 1 } Load 1 & & 2.10 \\
\cline { 1 - 1 } Load 2 & & 1.58 \\
\hline
\end{tabular}

The results for the different gimbal commands are not listed here since angles which are too small cannot give distinct restoration angle, whereas a too large one would make the load turn too much; exceeding testing angles. More experiments with various kinds of loads are needed to obtain the more precise characteristics line, but we present 3 points (small circles in the Figure 6) in this experiment.

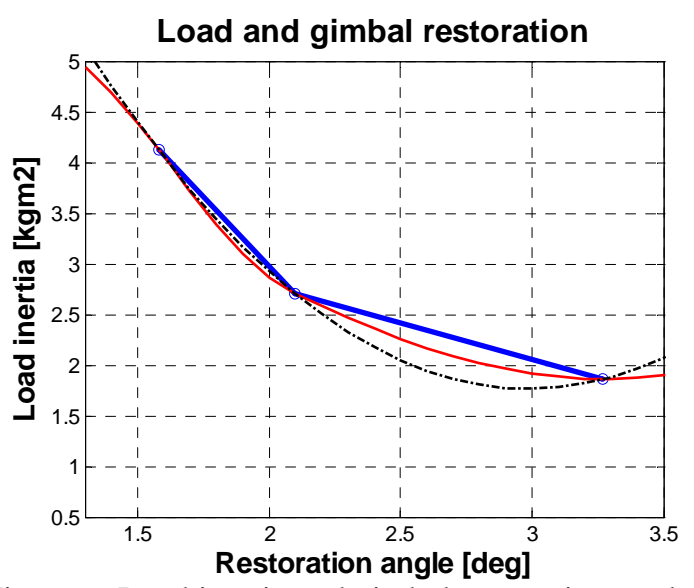

Figure 6. Load inertia and gimbal restoration angle 
It is possible to figure out the load if we measure the restoration angle by issuing test command before the actual command. To do this, we have to find the relationship function between load and restoration angle by the second order fitting equation to the results in Figure 6 (dashed line), as follows. This can be obtained using the Matlab function 'fit' with the fitting option 'pchipinterp' as shown in the figure (light solid line).

$$
\begin{aligned}
& y=a x^{2}+b x+c \\
& a=1.1910, b=-7.1137, c=12.3864
\end{aligned}
$$

The algorithm to implement this procedure, unknown load estimation, is as follows.

\section{STEP 1 : Detect peak value of the gimbal with given gimbal command.}

STEP 2 : Enter stabilizing mode by turning off the power of gimbal.

STEP 3 : Detect level value of the gimbal when load stops.

STEP 4 : Calculate restoration angle by subtracting the peak value with the level value.

STEP 5 : Find the inertia of the unknown load from the fitting equation.

\subsection{Load motion to gimbal command}

In the previous section, we could estimate the inertia of the unknown load. Now it is necessary to decide proper gimbal command angle for the load. To do this, figuring out the relationship between load and gimbal command angle is needed, which requires extensive experiment for many known loads at specific angular velocity of the loads, 12.5 [deg/sec]. The results are shown in Table 4 and some are illustrated in Figure 7.

Table 4. Gimbal restoration angle for loads

\begin{tabular}{|c|c|c|c|}
\hline Load & Inertia & $\begin{array}{c}\text { Restoration } \\
\text { angle }\end{array}$ & $\begin{array}{c}\text { Gimbal } \\
\text { command }\end{array}$ \\
\hline \hline Load 1 & 1.86 & 3.27 & 11 \\
\hline Load 2 & 2.70 & 2.10 & 14 \\
\hline Load 3 & 4.12 & 1.58 & 18 \\
\hline
\end{tabular}

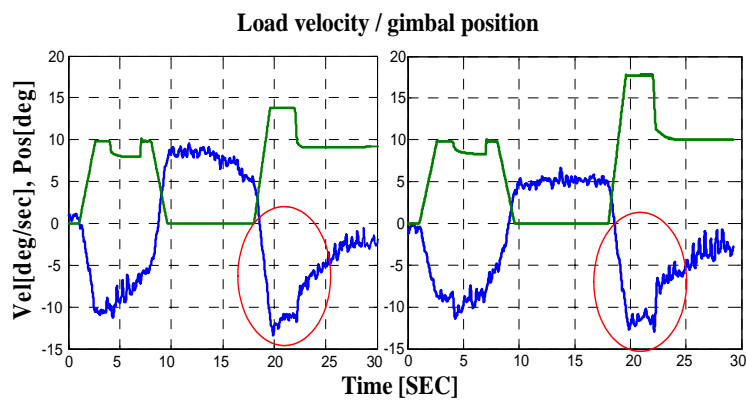

(a): Load 2 (b): Load 3

Figure 7 . The load angular velocity and gimbal

\section{trajectory}

The circled parts, in the figure, stand for the angular velocity of the load that we have to notice whereas the beginning parts are for the operation to obtain the restoration angles. The details of the gimbal operation are as following:

Operation 1: position command, 10 degree, at 1 second. Operation 2: motor power off at 4 second.

Operation 3: motor power on at 7 second.

Operation 4: position command, 0 degree, at 8 second.

Operation 5: position command, desired degree, at 18 second.

Operation 6: motor power off at 22 second.

The relationship between load and gimbal command angle is as in Figure 8. From this figure, we can find the relationship function between load and gimbal command angle by fitting an equation to the result. This can be obtained using the method used for the Figure 6.

$$
\begin{aligned}
& y=a x^{2}+b x+c \\
& a=-0.3339, b=5.0938, c=2.6805
\end{aligned}
$$

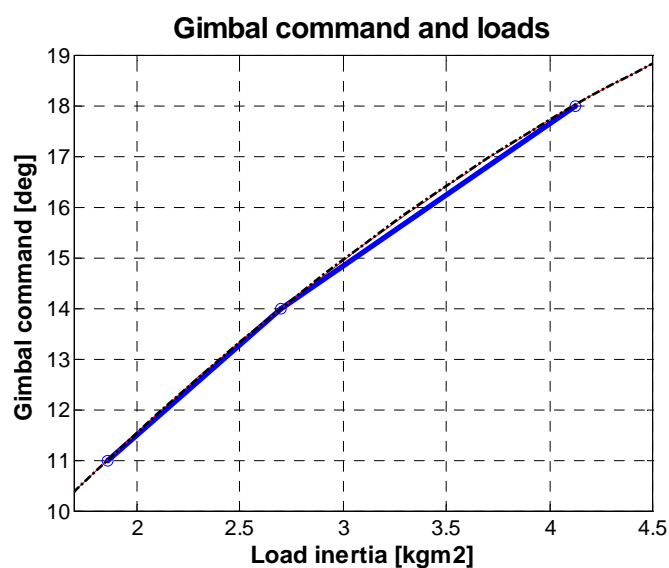

Figure 8. Gimbal command and loads

\subsection{Load control}

Following the procedures listed above, we demonstrate velocity control of an unknown load using the balance beam. We made unknown load by putting two stuffs on the frame hanging the load 1 shown in Table 2, which has inertia between load 1 and load 2 but it is not necessary to measure it because we have to deal with the unknown load. The target velocity is 12.5 [deg/sec].

We could have estimated the load to be 5.27 since we have the restoration angle 1.27 (A in the Figure 9), which came from the fitting equation (9) in Section 3.1 and is bigger than we expected. However, it seems reasonable because the gimbal command angle for this load, 20.25 in degree, is bigger than the angle of the load 3, 18.0 in degree.

The gimbal command angle was obtained from 
the fitting equation (10) in Section 3.2, which had been modified by the controller based on load had estimated.

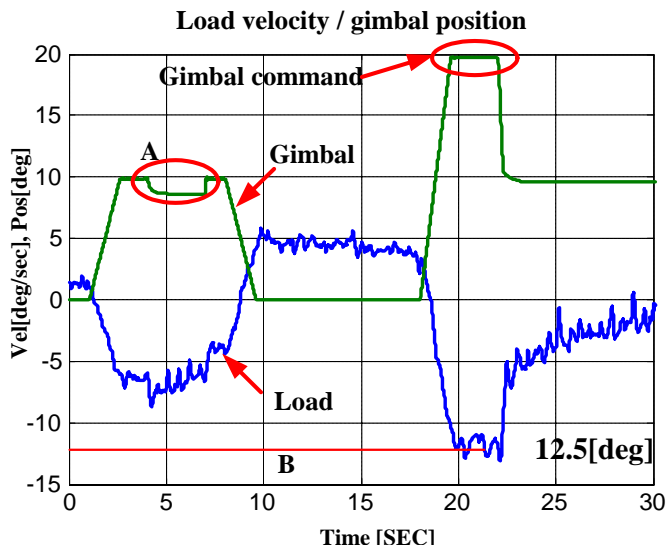

Figure 9. Angular velocity of unknown load and gimbal trajectory

In the figure, 'Load' stands for the angular velocity of the unknown load monitored by the gyro sensor, 'Gimbal' represents the angle of the gimbal. Note that the velocity of the unknown load is about 12.5 [deg/sec] as expected (B in the figure).

\section{CONCLUSION}

In this work, we designed a balance beam controller that can estimate the inertial of unknown load and control the angular velocity of it.

Unknown load could be estimated by investigating the characteristics of the restoration angle of the balance beam. We defined the fitting equation to estimate the load with the restoration angle measured. We also defined another fitting equation which can decide gimbal command angle by the estimated load. Finally, we could be able to implement the balance beam which can control the velocity of the unknown loads without an angular velocity sensor.

Thru an experiment we were able to show that the controller we developed is very convenient in handling construction work.

\section{REFERENCES}

[1] Vernon D. Barger, Martin G. Olsson, Classical Mechanics, McGRAW-HILL, 1995.

[2] J. L. Merian, L. G. Kraige, ENGINEERING MECHANICS, WILEY, 1993.

[3] Hiroshi Kanki, Yoshitsugu Nekomoto, Hiroyuki Monobe, Hironobu Ogura, Kiichi Kobayashi, "Development of CMG Active Vibration Control Device for Gondola," JSME Int. J. Series C, Vol. 37, No. 3, 1994.
[4] Jasim Ahmed, Robert H. Miller, Edward H. Hoop man, Vincent T. Coppola, Dennis S. Bernstein Tracie Andrusiak, David Action, "An Actively Controlled Control Moment Gyro/GyroPendulum Testbed,” Proc. 97 IEEE Int. Conf. on Control Applications. pp. 250-252, 1997.

[5] Feiyue Li, Peter M. Bainum, N. Glenn Creamer, Shalom Fisher, "Rapid Reorientation Maneuvers of Experimental Spacecraft with a Pendulum Appendage,” J. of Guidance Control \& Dynamics, V.21 N.1, pp. 164-171, 1998.

[6] Keon Young Yi, Young Gu Chung, "An Impleme ntation of a Gyro Actuator for the Attitude Control of an Unstructured Object," IEEE Int. Conf. Robotic and Automation., Vol. 2, pp. 1626 - 1631, 1999.

[7] Keon Young Yi, Man Oh Kwon, "An attitude control and stabilizatioin of an unstructured object using CMG subsystem," Proceeding of the ISIM 2000, Oct. 4-7, pp. 311-316, 2000.

[8] Chi-Tsong Chen, Analysis and Synthesis of Linear Control System, Pond Woods Press, 1978. 